\title{
Acylcarnitines: Can They Be Biomarkers of Diabetic Nephropathy?
}

\author{
Xiaodie Mu' ${ }^{1, *}$, Min Yang ${ }^{1} *$, Peiyao Ling ${ }^{1}, *$, Aihua Wu', Hua Zhou', Jingting Jiang ${ }^{2}$ \\ 'Department of Nephrology, The Third Affiliated Hospital of Soochow University, Changzhou, 213003, People's Republic of China; ${ }^{2}$ Department of \\ Tumor Biological Treatment, The Third Affiliated Hospital of Soochow University, Changzhou, 213003, People's Republic of China \\ *These authors contributed equally to this work
}

Correspondence: Hua Zhou; Jingting Jiang, Department of Nephrology, The Third Affiliated Hospital of Soochow University, Changzhou, 2I3003, People's Republic of China, Tel +86051968872082, Email zhouhua2323@suda.edu.cn; jiangjingting@suda.edu.cn

\begin{abstract}
Diabetic nephropathy (DN), one of the most serious microvascular complications of diabetes mellitus (DM), may progress to end-stage renal disease (ESRD). Current biochemical biomarkers, such as urinary albumin excretion rate (UAER), have limitations for early screening and monitoring of DN. Recent studies have identified some metabolites as candidate biomarkers for early detection of DN. In this review, we summarize the role of dysregulated acylcarnitines (AcylCNs) in DN pathophysiology. Lower abundance of short- and medium-chain AcylCNs and higher long-chain AcylCNs often occurred in DM with normal albuminuria and microalbuminuria, compared with advanced stages of DN. The increase of long-chain AcylCNs was supposed to be an adaptive compensation in fat acids (FAs) oxidation in the early stage of DN. Conversely, the decrease of longchain AcylCNs was due to incomplete oxidation of FAs in advanced stage of DN. Thus, AcylCNs may serve as sensitive biomarkers in predicting the risk of $\mathrm{DN}$.
\end{abstract}

Keywords: acylcarnitine, biomarkers, diabetic nephropathies, metabolomics, mitochondrial dysfunction

\section{Introduction}

The incidence of diabetes mellitus (DM) continues to rise worldwide. DM is one of the most important public health concerns with high rates of morbidity and mortality. ${ }^{1-3}$ Diabetic nephropathy (DN) is a severe complication of DM, which is a leading cause of end-stage renal disease (ESRD) in the developed world. ${ }^{4-7}$ Approximately $25-40 \%$ of DM patients develop DN. ${ }^{8}$ Accordingly, an urgent need exists for early biomarkers to predict DN among individuals with DM.

DN initially causes glomerular hyperfiltration, followed by varying degrees of proteinuria, decline in glomerular filtration rate (GFR), and finally ESRD. ${ }^{9,10}$ Although the etiology of DN is not clear, it is mainly due to abnormal glucose metabolism, oxidative stress, activation of innate immune system, and inflammation. ${ }^{1-14}$ It is generally believed that, once developed, DN is difficult to be reversed. In advanced DN, dialysis or kidney transplant is needed for survival. ${ }^{15}$ However, the clinical manifestations of DN are often silent in the early stage. ${ }^{16}$ Urinary albumin excretion rate (UAER) is considered to be an early marker of DN. ${ }^{17}$ However, the levels of UAER are not increased in stages I and II of DN. ${ }^{13,18,19}$ In addition, renal biopsy is considered the gold standard for diagnosis and determines the degree of $\mathrm{DN}^{20}$ However, it is invasive and expensive, and its results do not correlate with the clinical outcome. ${ }^{21}$ Therefore, a promising clinical marker for early diagnosis of DN is urgently needed. DM, a metabolic disease defined by chronic elevation of glucose, leads to downstream metabolic dysfunction. Metabolomics is the study of small endogenous molecules from cells and biological systems. The changes of urine or plasma metabolites may predict the development of DN in DM individuals. Among multiple metabolites, acylcarnitines (AcylCNs) highlight unique features in DN. This review emphasizes on the role of AcylCNs in DN, and acting as early predictive biomarkers of DN. 


\section{Graphical Abstract}

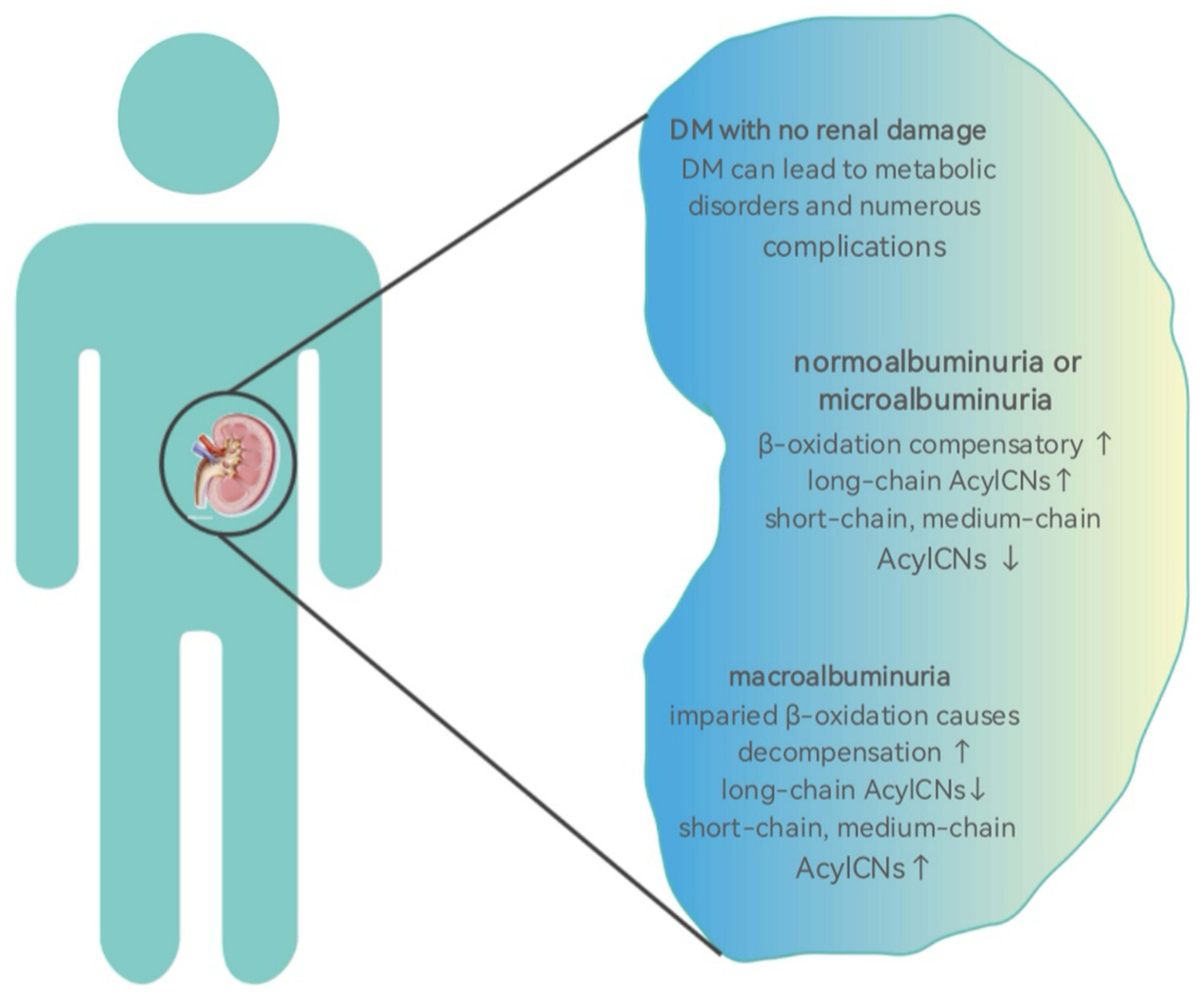

\section{Metabolomics in DN}

Metabolomics is a qualitative and quantitative analysis of metabolites in organisms after environmental stimuli or gene alteration, which can be used to identify metabolites closely related to physiological and pathological changes. ${ }^{22}$ Metabolomics can reveal the metabolites that may be specific to disease and play an important role in the development of disease. ${ }^{22,23}$ Metabolomics techniques, such as nuclear magnetic resonance (NMR) and mass spectrometry (MS), have been applied to the urine and circulating metabolites in DN in a targeted or untargeted manner. ${ }^{24}$ Targeted metabolomics, the gold standard for metabolite quantification, is to detect only a few groups of metabolites and obtain high sensitivity and selectivity by excluding a large number of mixed signals. It has been used to identify biomarkers for DN. ${ }^{25-27}$ By using targeted metabolomics, Zhang et al found a variety of early metabolic signs in DN and identified 11 closely related metabolites, which helped to further predict and prevent DN in the Chinese population. ${ }^{28}$ Jiang et al used highperformance liquid chromatography-electrospray ionization tandem MS (HPLC-ESI-MS/MS) to simultaneously quantify eight aminothiols in the metabolic cycle of homocysteine in plasma and identified two sulfur-containing metabolites, namely S-adenosylmethionine and S-adenosylhomocysteine, as potential biomarkers for DN. ${ }^{29}$ Researchers found that AcylCNs could help identify individuals with proteinuria or renal dysfunction based on novel targeted metabolomics approaches, suggesting the increased importance of AcylCNs as biomarkers of DN. They could improve the predictive capacity of clinical models to identify kidney dysfunction and DN-related outcomes. ${ }^{30}$

The establishment of sensitive biomarkers for DN may help discover novel therapeutic targets. ${ }^{31}$ Metabolic change in DM patients may be triggers for DN. Previous studies have found that amino acids (AAs), fatty acids (FAs), purines, pyrimidines, and other metabolites were altered in the urine and peripheral blood circulation in DN (Table 1). ${ }^{32-37}$ Metabolomics, especially targeted metabolomics, has been recognized as a powerful tool in the field of biomarker discovery of DN. 
Table I Related Studies of Metabolomics in DN

\begin{tabular}{|c|c|c|c|c|}
\hline Material & Sample & Method & Major Finding & Reference \\
\hline Serum & Human & LC-MS & Tryptophan as a surrogate prognostic marker for DN & [38] \\
\hline Serum & Human & LC-MS/MS & Taurine metabolism pathway potentially affect the pathogenesis of DN from T2DM & [20] \\
\hline Urine & Human & NMR & Identify novel metabolic difference between $\mathrm{DN}$ and non-DN & [39] \\
\hline Serum & Human & LC-MS/MS & Elevated tyrosine is associated with increased DN risk & [40] \\
\hline Plasma & Rat & GC-MS & Oleic acid might be the potential biomarker of kidney injury & {$[4 I]$} \\
\hline $\begin{array}{l}\text { Kidney } \\
\text { tissue }\end{array}$ & Rat & LC-MS & Mitochondrial ceramide accumulation may result in podocyte damage & [42] \\
\hline Plasma & Human & $\begin{array}{l}\text { UHPLC- } \\
\text { QTOF/MS }\end{array}$ & $\begin{array}{l}\text { Sphingomyelin and phosphatidylcholine species are associated with renal impairment and } \\
\text { all-cause mortality in TIDM }\end{array}$ & [43] \\
\hline Serum & Human & $\begin{array}{l}\text { UPLC-ESI- } \\
\text { MS/MS }\end{array}$ & $\begin{array}{l}\text { Linolelaidic Acid, L-Dihydroorotic Acid, and Azoxystrobin Acid especially represented } \\
\text { a potential indicator of DM progress }\end{array}$ & [28] \\
\hline $\begin{array}{l}\text { Kidney } \\
\text { tissue }\end{array}$ & Rat & LC-MS & Linoleic acid metabolism and fatty acids $\beta$-oxidation are inhibited in DN & [44] \\
\hline Plasma & Human & MS & Increased acylcarnitines levels are associated with risk of progression to ESRD & [45] \\
\hline Blood & Rat & $\begin{array}{l}\text { SPE-UPLC- } \\
\text { MS/MS }\end{array}$ & A correlation between metabolic disorders of purine and pyrimidine and DN & {$[25]$} \\
\hline Urine & Human & LC-MS & $\begin{array}{l}\text { Xanthosine and NI-methylguanosine can be used to predict nephropathy in patients with } \\
\text { T2DM }\end{array}$ & [46] \\
\hline Plasma & Human & LC-MS/MS & $\begin{array}{l}\text { Increased lipoxygenase metabolites and decreased CYP450 metabolites are significantly } \\
\text { associated with the incidence of DN }\end{array}$ & [47] \\
\hline Serum & Human & GC/LC-MS & Some modified metabolites were associated with renal function decline and time to ESRD & [48] \\
\hline
\end{tabular}

Notes: The above table lists some recent studies on metabolomics of DN. In the process of diabetic kidney damage, the metabolic disorders of various substances appear in the body. It is not difficult to find that researchers have begun to focus on metabolomics of DN in recent years, so as to further clarify the mechanism of early diabetic renal damage, which may help to identify preventive and treatment measures for DN.

Abbreviations: DN, diabetic nephropathy; DM, diabetes mellitus; ESRD, end-stage renal disease; UAER, urinary albumin excretion rate; AcylCNs, acylcarnitines; FA, fat acid; GFR, glomerular filtration rate; NMR, nuclear magnetic resonance; MS, mass spectrometry; HPLC-ESI-MS/MS, high performance liquid chromatography-electrospray ionization tandem mass spectrometry; AAs, amino acids; OXPHOS, oxidative phosphorylation; TCA, tricarboxylic acid; CN, carnitine; MS/MS, tandem mass spectrometry; C2, acetyl-CoA; T2DM, type 2 diabetes mellitus; eGFR, estimated glomerular filtration rate; TIDM, type I diabetes mellitus; CKD, chronic kidney disease; ACC, acetyl-CoA carboxylase; ACR, albumin/creatinine ratio.

\section{AcyICNs-Potential Biomarkers of DN}

It is suggested that mitochondrial dysfunction plays an indispensable role in DN. ${ }^{49,50}$ Mitochondria, known as powerhouse of the cell, are the basic subcellular organelles in eukaryotic cells to maintain metabolic homeostasis. Neurodegenerative, neoplastic, endocrine, and cardiovascular diseases are associated with mitochondrial dysfunction. ${ }^{37,51}$ Mitochondria are essential for ATP synthesis via oxidative phosphorylation (OXPHOS) ${ }^{52}$ The number of mitochondria in the kidneys is second only to the heart. Tricarboxylic acid (TCA) cycle, the hub of three nutrients, is carried out in mitochondria. ${ }^{53,54}$ Therefore, dysfunction of the TCA cycle causes metabolic disorders of AAs, FAs, and purines (Figure 1). Previous studies have showed that mitochondrial dysfunction in DN causes $\beta$-oxidation of FA disorder, ultimately leading to abnormal deposition of lipids in $\mathrm{DN} .{ }^{44,53}$ AcylCNs serve as markers of mitochondrial function, which play a critical role in the pathogenesis of DN, specifically for $\beta$-oxidation of FA. ${ }^{55}$

\section{Metabolic Process Related to AcylCNs in Organisms}

AcylCNs contain an acyl esterified to carnitine $(\mathrm{CN})$, which allows long-chain FAs to pass through the mitochondrial membrane for FA $\beta$-oxidation. ${ }^{56-58}$ AcylCNs, intermediate metabolites in FA and AA oxidation, are located in tissues and body fluids. Tandem mass spectrometry (MS/MS) detection of AAs and AcylCNs has been widely used for screening neonatal metabolic diseases. ${ }^{5,59,60}$ AcylCNs are synthesized by carnitine palmitoyl transferase I, which is responsible for transporting FAs to the mitochondrial matrix. AcylCNs enter the mitochondria where they are subjected to carnitine palmitoyl transferase II in the inner mitochondrial membrane, which transforms them to acyl-CoA and releases carnitine. ${ }^{61}$ Figure 2 shows the metabolic pathway for production of AcylCNs. Medium-chain and long-chain acyl- 


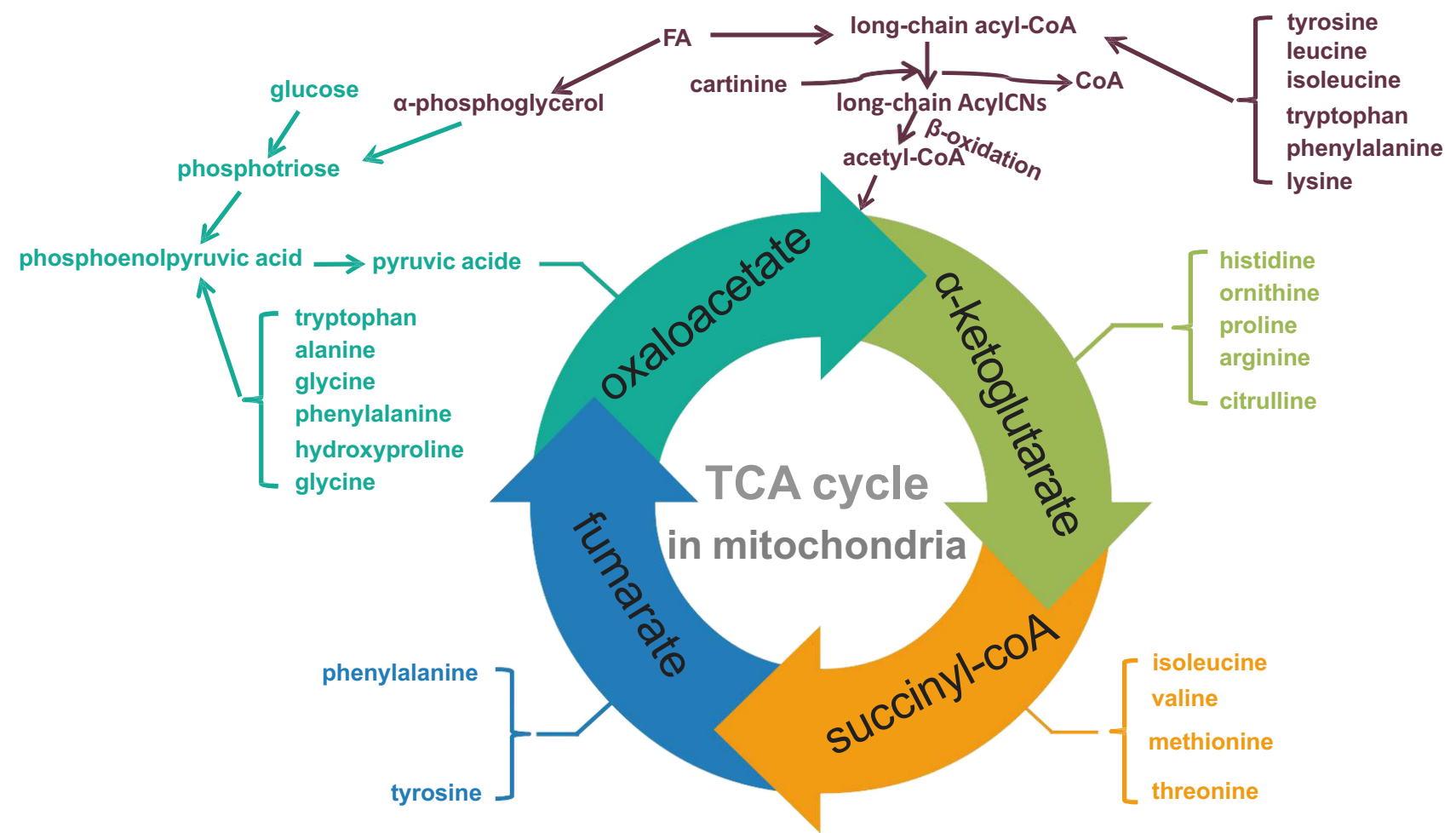

Figure I Tricarboxylic acid cycle-three metabolic hubs.

Notes: TCA cycle is the common pathway for the decomposition capacity of the three major nutritional metabolites (glucose, FA and AA), also is the hub of their metabolism. TCA cycle needs to be carried out in mitochondria. When DN causes mitochondrial dysfunction and AGEs increase, it is bound to cause metabolic disorders. Abbreviations: TCA cycle, tricarboxylic acid cycle; FA, fatty acid; AA, amino acid; AGEs, advanced glycation end products.

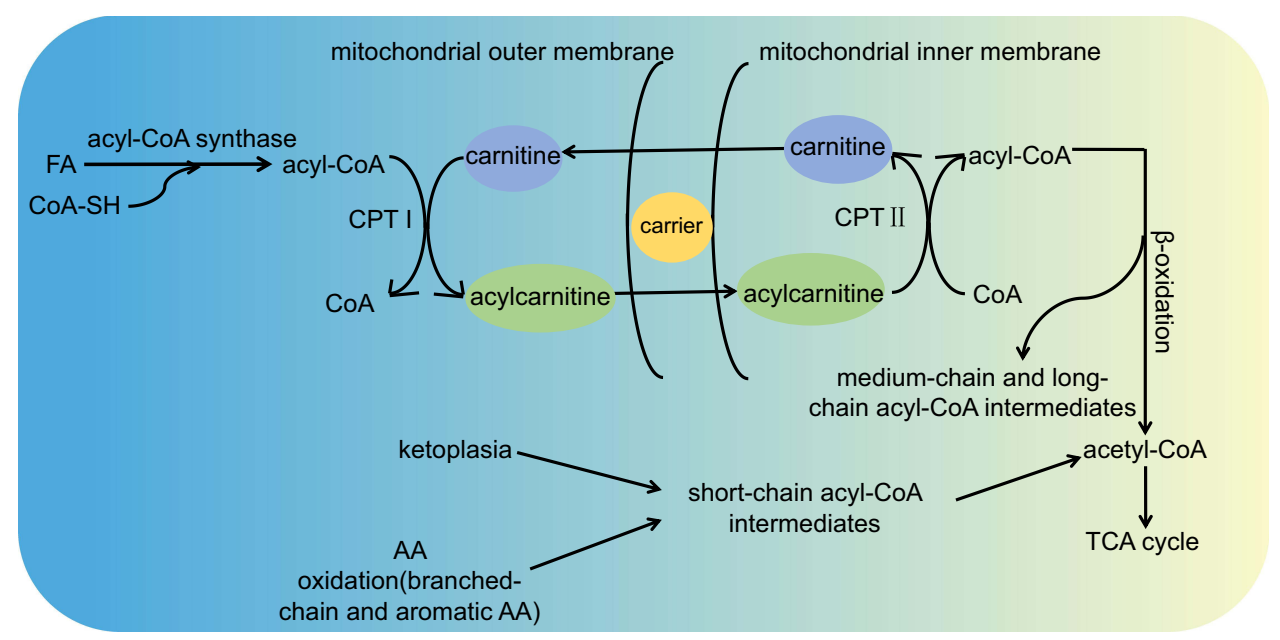

Figure 2 The metabolic process of AcylCNs in organisms.

Notes: When $\beta$-oxidation or AA oxidation is incomplete, or ketone body formation is insufficient, acyl-CoA intermediates accumulate and produce less C2. Due to the cytotoxicity of excess acyl-CoA, they are converted into AcylCNs and released into the plasma, eventually excreted into the urine. AcylCNs are important markers of peroxisome and mitochondrial oxidative disorder, which can serve as biomarkers for the metabolic syndrome.

Abbreviations: AcylCNs, acylcarnitines; TCA cycle, tricarboxylic acid cycle; FA, fatty acid; AA, amino acid; C2, acetyl-CoA; CPT I, carnitine palmitoyl transferase I; CPT II, carnitine palmitoyl transferase II.

CoA intermediates are generated during FA oxidation, whereas short-chain acyl-CoA intermediates are generated during AA oxidation (branched amino acids and aromatic amino acids) and ketone body formation. ${ }^{62,63}$ During the active oxidation of FAs and AAs, acyl-CoA intermediates are produced, which are immediately converted into short products 
and acetyl-CoA (C2), thus entering the TCA cycle. ${ }^{64}$ With incomplete oxidation or insufficient ketone body formation, acyl-CoA intermediates accumulate and produce less C2. Due to the cytotoxicity of excess acyl-CoA, they are converted to AcylCNs, released into plasma, and eventually excreted into urine. ${ }^{65}$

AcylCNs are important markers of peroxisome and mitochondrial oxidative disorders and can serve as biomarkers for metabolic syndrome. ${ }^{66-71}$ Some scholars used targeted metabolomics to study a group of AcylCNs, evaluated their relationship with the incidence of DM, and incorporated them into the established DM risk model. ${ }^{72}$ The results showed the accuracy and applicability of the model improved when added metabolomic data. In addition, some related literature indicated that AcylCNs were related to type 2 DM (T2DM), suggesting that AcylCNs accumulation could cause imbalance of insulin synthesis and secretion, which further led to $\beta$ cell dysfunction. ${ }^{73,74}$ Moreover, researchers highlighted the importance of AcylCNs as biomarkers in clinical predictive models associated with rapid estimated GFR (eGFR) decline in type $1 \mathrm{DM}$ (T1DM) combined with traditional risk factors. ${ }^{75}$ The study of AcylCNs metabolic spectrum is of great significance for understanding the occurrence of DM and its complications. ${ }^{76-78}$ Plasma AcylCNs were also significantly associated with prognosis and treatment response in IgA nephropathy patients. Baseline levels of AcylCNs were associated with 1-year eGFR decline. ${ }^{79}$ In different stages of chronic kidney disease (CKD), the abundance of AcylCNs was constantly fluctuating, and the ratio of long-to-intermediate AcylCNs was gradually decreasing as the kidney disease progresses. The trend of long-to-intermediate AcylCNs ratio decreased gradually from CKD stage 2 to $5 .{ }^{80}$ It was supposed that the early increase of FA oxidation may be an adaptive phenomenon during the progression of CKD to cope with the elevated saturated FA, but impaired $\beta$-oxidation continued to prevail as the disease progressed and formed a vicious circle. In this case, impaired oxidation caused intracellular FA accumulation, further impaired mitochondria. ${ }^{80}$ Based on the above studies, AcylCNs are considered to be important metabolites of DM and DM complications.

\section{Metabolic Changes in AcylCNs in DN}

The study of lipidomics revealed the potential mechanisms underlying the development of DN: impaired mitochondrial $\beta$-oxidation and complex lipid remodeling. ${ }^{55,81}$ The concentrations of carnitine, a carrier of FA transport into the mitochondria, and AcylCNs indicate the rate of $\beta$-oxidation of FA in the progression of $\mathrm{DN} .{ }^{82} \mathrm{DN}$ progression is associated with disorders of FA synthesis, desaturation, and oxidation. AcylCNs can accumulate in the plasma due to mitochondrial damage, especially in individuals with reduced eGFR. ${ }^{30,83}$ Previous study suggested that the increased levels of AcylCNs in urine were associated with early kidney injury in DM patients, reflecting the changes in $\beta$-oxidation pathway. ${ }^{84}$ Theoretically, all short-, medium-, and long-chain AcylCNs would be elevated in patients with DN. Actually, the concentrations of AcylCNs in different lengths varied between different stages of DN. The plasma short- and medium-chain AcylCNs were increased, whereas long-chain AcylCNs were decreased in DM patients with normoalbuminuria and microalbuminuria, while the plasma short- and medium-chain AcylCNs were decreased, and the long-chain AcylCNs were increased. ${ }^{85}$ A possible explanation is that FA oxidation may cause adaptive compensation (FA prolongation and desaturation) in DM with normoalbuminuria and microalbuminuria. ${ }^{55}$ It converts more toxic lipids (saturated nonesterified FA) into less toxic lipids (polyunsaturated triglycerides) to prevent kidney injury, rather than reducing the utilization of these FA intermediates for mitochondrial oxidation. When DM patients progress to macroalbuminuria, mitochondrial oxidation in the kidneys is impaired in a state of decompensation. It is difficult to compensate for the renal metabolism and pathological changes by strengthening FA oxidation. Thus, the incomplete $\beta$-oxidation of long-chain FAs is increased, resulting in an increase in AcylCNs intermediates of plasma short- and medium-chain AcylCNs. What is more, lipid metabolic changes associated with DM are reported to occur at the early stage of DN. Studies of lipid metabolism can help discriminate between progression and non-progression patients and allow risk stratification in DM patients. ${ }^{55}$ In addition, previous study demonstrated that the activation of acetyl-CoA carboxylase (ACC) was a key determinant of DN progression. ${ }^{85}$ Upregulation of ACC suppresses CTPI, which in turn suppresses the cytoplasmic transformation of long-chain acyl-CoA to long-chain AcylCNs, thereby reducing the substrate of carnitine shuttle, impairing $\beta$ - oxidation of long-chain FAs, and increasing the abundance of cytoplasmic palmitic acid. Long-chain AcylCNs are an important substrate for $\beta$-oxidation, so the $\beta$-oxidation is downregulated accordingly (Figure 3 ). Interestingly, the short-chain AcylCNs (C4, C4-OH, C5:1, C5-OH, and C5DC) were observed to be associated with 


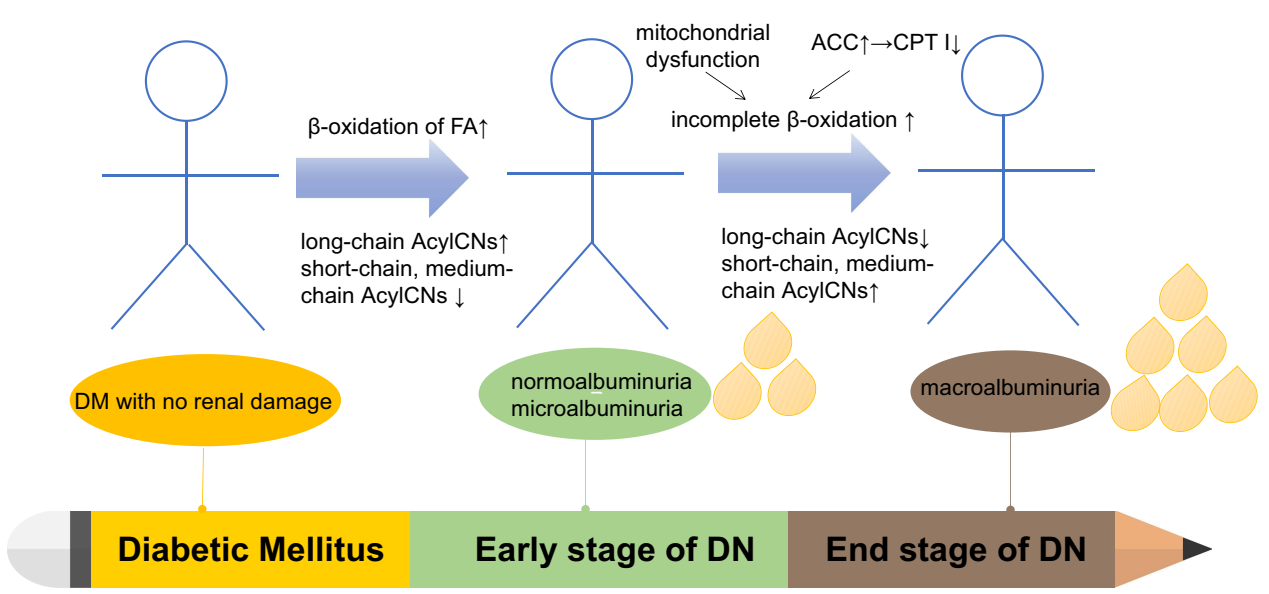

Figure 3 AcylCNs in DN.

Notes: The abundance of AcylCNs have different changes during the progression from DM to DN, even including AcylCNs with different length chains. These changes are mainly due to the increase of long-chain AcyICNs caused by adaptive compensation enhancement in early FA oxidation, on the contrary, the decrease caused by incomplete oxidation enhancement of FAs caused by mitochondrial dysfunction in the late stage. Drips represent the amount of protein in urine.

Abbreviations: AcylCNs, acylcarnitines; DM, diabetic mellitus; DN, diabetic nephropathy; FAs, fatty acids; ACC, acetyl-CoA carboxylase; CPT I, carnitine palmitoyl transferase I.

urinary microalbumin levels. In particular, C4 was a positive, independent, and significant predictor of the albumin/ creatinine ratio (ACR) levels. ${ }^{33}$ During the progression from normoalbuminuria to microalbuminuria, an abundance of C4 entered to the TCA cycle, thereby inhibiting glucose oxidation, exacerbating intracellular demand for carnitine storage. C4 concentration in the plasma was significantly elevated when DM progressed from normoalbuminuria to microalbuminuria. In summary, when stratified according to the levels of albuminuria, patients with normoalbuminuria and microalbuminuria have higher levels of long-chain AcylCNs than those with macroalbuminuria accompanied by $\beta$ oxidation activated. As DN progresses, the levels of long-chain AcylCNs reduced as a result of downregulated $\beta$ oxidation.

\section{Limitations and Future Perspective of AcylCNs in DN}

Metabolomics is the science of measuring small molecules in body fluids, which can probe the biomarkers to understand the pathogenic mechanisms and discover the novel therapeutic targets. ${ }^{23,86,87}$ However, the application of metabolomics has certain limitations, and there are some challenges when AcylCNs serve as biomarkers of DN. First, the metabolic products change in organisms in different nutritional status. ${ }^{88,89}$ It is difficult to confirm that all DM patients have the same dietary conditions. Different lipid diets interfere with changes in AcylCNs concentrations in blood and urine. So, are AcylCNs still reliable as indicators of kidney injury in DM patients with different diet habits? However, in fact, most DM patients have a similar diet when included in the diet management of DM. AcylCNs are not significantly disturbed by the confounding factors of the diet habits. Of course, further studies are needed to confirm whether diet influences the AcylCNs concentrations in DN patients. Meanwhile, we suggest that AcylCNs combined with traditional indicators such as UAER, ACR, eGFR, and even renal biopsy may achieve more precise prediction of DN. Second, the baseline AcylCNs levels in different age groups are actually different. ${ }^{90}$ If we simply judge the AcylCNs levels with a constant standard, it is actually unreasonable. We suggest that the metabolic spectrum of AcylCNs should be standard by age. Finally, the clinical significance of AcylCNs is still not confirmed, and the mechanisms of AcylCNs involved in DN pathogenesis and progression need more studies in the future. ${ }^{22}$ The abundance of AcylCNs is not only influenced by the production but also by consumption in a nonlinear manner. ${ }^{88}$ It was proposed that isotope tracers combined with metabolomics can reveal the pathway activities to evaluate AcylCNs concentrations. Recent advances in neural network (computer-based prediction method) to metabolomics is promising. ${ }^{91}$ It may help to predict the biological activity of AcylCNs in DN progression. 
Metabolomics is a promising diagnostic tool in early and pre-symptomatic onset of disease and may supply personalized treatment. For example, previous studies have found that the addition of leucine to the diet reduced insulin resistance, interfered with TCA circulating metabolism, and decreased urinary carnitine levels in $\mathrm{db} / \mathrm{db}$ mice by urine metabolomics. Furthermore, in these mice, autophagy was increased in the renal cortex, and DN was improved. ${ }^{92}$ Metabolomics can identify biomarkers or genetic phenotypes associated with pathophysiology by regulating the levels of the genome, epigenome, transcriptome, and proteome, which help to intervene by dietary supplementation of an abnormal metabolite or knockout gene phenotype. ${ }^{93,94}$ Although there is no relevant literature to support the supplement of long-chain AcylCNs to relieve DN, it may be a promising therapeutic target in the future. Targeted metabolomics shed light on the early detection of DN and unveiling potential biomarkers.

\section{Conclusion}

$\mathrm{DN}$, as well as its onset and progression, is often insidious. UAER and renal biopsy have limitations for the early and accurate diagnosis. The emergence of metabolomics provides insight into DM kidney injury in a non-invasive manner. Based on novel targeted metabolomics strategies, we can identify plasma and urine biomarkers (including AcylCNs) together to elucidate the mechanisms and establish a clinical prediction model. ${ }^{30,72}$ This review mainly describes the changes in AcylCNs in the lipid in DN. Lower abundance of short- and medium-chain AcylCNs, and higher long-chain AcylCNs occur in DM with normal albuminuria and microalbuminuria, while higher abundance of short-chain and medium-chain AcylCNs and lower long-chain AcylCNs occur in DM with macroalbuminuria. Further research is needed to understand the changes, pathogenesis, and the clinical significance of AcylCNs involved in DN. It is recommended to use isotope tracer or neural network combined with targeted metabolomics to quantify AcylCNs of different lengths and the metabolic pathway in DN. Future studies are needed to determine the threshold values of AcylCNs in plasma or urine to predict DN. In summary, AcylCNs are promising biomarkers for early diagnosis of DN.

\section{Acknowledgments}

Financial Support: This work was supported by grants from the National Natural Science Foundation of China (82000684) and Changzhou Sci \& Tech Program (CJ20200025).

Authors' contributions: All authors contributed to drafting or revising the article, have agreed on the journal to which the article will be submitted, gave final approval of the version to be published, and agree to be accountable for all aspects of the work.

\section{Disclosure}

The authors report no conflicts of interest in this work.

\section{References}

1. King H, Aubert RE, Herman WH. Global burden of diabetes, 1995-2025: prevalence, numerical estimates, and projections. Diabetes Care. 1998;21 (9):1414-1431. doi:10.2337/diacare.21.9.1414

2. Cho NH, Shaw JE, Karuranga S, et al. IDF diabetes atlas: global estimates of diabetes prevalence for 2017 and projections for 2045. Diabetes Res Clin Pract. 2018;138:271-281. doi:10.1016/j.diabres.2018.02.023

3. Ogurtsova K, da Rocha Fernandes JD, Huang Y, et al. IDF diabetes atlas: global estimates for the prevalence of diabetes for 2015 and 2040. Diabetes Res Clin Pract. 2017;128:40-50. doi:10.1016/j.diabres.2017.03.024

4. Brownlee M. Biochemistry and molecular cell biology of diabetic complications. Nature. 2001;414(6865):813-820. doi:10.1038/414813a

5. Colhoun HM, Marcovecchio ML. Biomarkers of diabetic kidney disease. Diabetologia. 2018;61(5):996-1011. doi:10.1007/s00125-018-4567-5

6. Hathaway CK, Chang AS, Grant R, et al. High Elmo1 expression aggravates and low Elmo1 expression prevents diabetic nephropathy. Proc Natl Acad Sci U S A. 2016;113(8):2218-2222. doi:10.1073/pnas.1600511113

7. Tuttle KR, Bakris GL, Bilous RW, et al. Diabetic kidney disease: a report from an ADA consensus conference. Diabetes Care. 2014;37 (10):2864-2883. doi:10.2337/dc14-1296

8. Barrios C, Spector TD, Menni C. Blood, urine and faecal metabolite profiles in the study of adult renal disease. Arch Biochem Biophys. 2016;589:81-92. doi:10.1016/j.abb.2015.10.006

9. Alicic RZ, Rooney MT, Tuttle KR. Diabetic kidney disease: challenges, progress, and possibilities. Clin J Am Soc Nephrol. 2017;12 (12):2032-2045. doi:10.2215/CJN.11491116

10. Nishad R, Tahaseen V, Kavvuri R, et al. Advanced-glycation end-products induce podocyte injury and contribute to proteinuria. Front Med (Lausanne). 2021;8:685447. doi:10.3389/fmed.2021.685447 
11. Gong P, Chang X, Chen X, et al. Metabolomics study of cadmium-induced diabetic nephropathy and protective effect of caffeic acid phenethyl ester using UPLC-Q-TOF-MS combined with pattern recognition. Environ Toxicol Pharmacol. 2017;54:80-92. doi:10.1016/j.etap.2017.06.021

12. Tang SCW, Yiu WH. Innate immunity in diabetic kidney disease. Nat Rev Nephrol. 2020;16(4):206-222. doi:10.1038/s41581-019-0234-4

13. Tonneijck L, Muskiet MH, Smits MM, et al. Glomerular hyperfiltration in diabetes: mechanisms, clinical significance, and treatment. $J$ Am Soc Nephrol. 2017;28(4):1023-1039. doi:10.1681/ASN.2016060666

14. Wei T, Shu Q, Ning J, et al. The protective effect of basic fibroblast growth factor on diabetic nephropathy through remodeling metabolic phenotype and suppressing oxidative stress in mice. Front Pharmacol. 2020;11:66. doi:10.3389/fphar.2020.00066

15. Cole JB, Florez JC. Genetics of diabetes mellitus and diabetes complications. Nat Rev Nephrol. 2020;16(7):377-390. doi:10.1038/s41581-020-0278-5

16. Nilavan E, Sundar S, Shenbagamoorthy M, Narayanan H, Nandagopal B, Srinivasan R. Identification of biomarkers for early diagnosis of diabetic nephropathy disease using direct flow through mass spectrometry. Diabetes Metab Syndr. 2020;14(6):2073-2078. doi:10.1016/j.dsx.2020.10.017

17. Remuzzi G, Schieppati A, Ruggenenti P. Nephropathy in patients with type 2 diabetes. $N$ Engl J Med. 2002;346(15):1145-1151. doi:10.1056/ NEJMcp011773

18. Di Bonito P, Mozzillo E, Rosanio FM, et al. Albuminuric and non-albuminuric reduced eGFR phenotypes in youth with type 1 diabetes: factors associated with cardiometabolic risk. Nutr Metab Cardiovasc Dis. 2021;31(7):2033-2041. doi:10.1016/j.numecd.2021.03.019

19. Panagiotopoulos S, Cooper ME, Jerums G. Progressive decline in renal function in diabetic patients with and without albuminuria. Diabetes. 1994;43(5):649-655. doi:10.2337/diab.43.5.649

20. Tao S, Zheng W, Liu Y, et al. Analysis of serum metabolomics among biopsy-proven diabetic nephropathy, type 2 diabetes mellitus and healthy controls. RSC Adv. 2019;9(33):18713-18719. doi:10.1039/C9RA01561B

21. Luciano RL, Moeckel GW. Update on the native kidney biopsy: core curriculum 2019. Am J Kidney Dis. 2019;73(3):404-415. doi:10.1053/j. ajkd.2018.10.011

22. Johnson CH, Ivanisevic J, Siuzdak G. Metabolomics: beyond biomarkers and towards mechanisms. Nat Rev Mol Cell Biol. 2016;17(7):451-459. doi: $10.1038 / \mathrm{nrm} .2016 .25$

23. Chen X, Shi BL, Qi RZ, Chang X, Zheng HG. Ultra-performance liquid chromatography/mass spectrometry-based metabolomics for discovering potential biomarkers and metabolic pathways of colorectal cancer in mouse model (ApcMin/+) and revealing the effect of honokiol. Front Oncol. 2021;11:671014. doi:10.3389/fonc.2021.671014

24. Khodadadi M, Pourfarzam M. A review of strategies for untargeted urinary metabolomic analysis using gas chromatography-mass spectrometry. Metabolomics. 2020;16(6):66. doi:10.1007/s11306-020-01687-x

25. Liu C, Gu C, Huang W, Sheng X, Du J, Li Y. Targeted UPLC-MS/MS high-throughput metabolomics approach to assess the purine and pyrimidine metabolism. J Chromatogr B Analyt Technol Biomed Life Sci. 2019;1113:98-106. doi:10.1016/j.jchromb.2019.03.008

26. Zhou J, Liu H, Liu Y, Liu J, Zhao X, Yin Y. Development and evaluation of a parallel reaction monitoring strategy for large-scale targeted metabolomics quantification. Anal Chem. 2016;88(8):4478-4486. doi:10.1021/acs.analchem.6b00355

27. Zhou J, Yin Y. Strategies for large-scale targeted metabolomics quantification by liquid chromatography-mass spectrometry. Analyst. 2016;141 (23):6362-6373. doi:10.1039/C6AN01753C

28. Zhang H, Zuo JJ, Dong SS, et al. Identification of potential serum metabolic biomarkers of diabetic kidney disease: a widely targeted metabolomics study. J Diabetes Res. 2020;2020:3049098. doi:10.1155/2020/3049098

29. Jiang Z, Liang Q, Luo G, Hu P, Li P, Wang Y. HPLC-electrospray tandem mass spectrometry for simultaneous quantitation of eight plasma aminothiols: application to studies of diabetic nephropathy. Talanta. 2009;77(4):1279-1284. doi:10.1016/j.talanta.2008.08.031

30. Ibarra-Gonzalez I, Cruz-Bautista I, Bello-Chavolla OY, et al. Optimization of kidney dysfunction prediction in diabetic kidney disease using targeted metabolomics. Acta Diabetol. 2018;55(11):1151-1161. doi:10.1007/s00592-018-1213-0

31. Fan Y, Li Y, Chen Y, et al. Comprehensive metabolomic characterization of coronary artery diseases. J Am Coll Cardiol. 2016;68(12):1281-1293. doi:10.1016/j.jacc.2016.06.044

32. Benito S, Sanchez-Ortega A, Unceta N, et al. Untargeted metabolomics for plasma biomarker discovery for early chronic kidney disease diagnosis in pediatric patients using LC-QTOF-MS. Analyst. 2018;143(18):4448-4458. doi:10.1039/C8AN00864G

33. Liu JJ, Ghosh S, Kovalik JP, et al. Profiling of plasma metabolites suggests altered mitochondrial fuel usage and remodeling of sphingolipid metabolism in individuals with type 2 diabetes and kidney disease. Kidney Int Rep. 2017;2(3):470-480. doi:10.1016/j.ekir.2016.12.003

34. Nishi H, Yamanaka D, Kamei H, et al. Importance of serum amino acid profile for induction of hepatic steatosis under protein malnutrition. Sci Rep. 2018;8(1):5461. doi:10.1038/s41598-018-23640-8

35. Rotbain Curovic V, Hansen TW, Eickhoff MK, et al. Urinary tubular biomarkers as predictors of kidney function decline, cardiovascular events and mortality in microalbuminuric type 2 diabetic patients. Acta Diabetol. 2018;55(11):1143-1150. doi:10.1007/s00592-018-1205-0

36. Tan SM, Ziemann M, Thallas-Bonke V, et al. Complement C5a induces renal injury in diabetic kidney disease by disrupting mitochondrial metabolic agility. Diabetes. 2020;69(1):83-98. doi:10.2337/db19-0043

37. Xiao L, Xu X, Zhang F, et al. The mitochondria-targeted antioxidant MitoQ ameliorated tubular injury mediated by mitophagy in diabetic kidney disease via Nrf2/PINK1. Redox Biol. 2017;11:297-311. doi:10.1016/j.redox.2016.12.022

38. Chou CA, Lin CN, Chiu DT, Chen IW, Chen ST. Tryptophan as a surrogate prognostic marker for diabetic nephropathy. J Diabetes Investig. 2018;9 (2):366-374. doi:10.1111/jdi.12707

39. Barrios C, Zierer J, Wurtz P, et al. Circulating metabolic biomarkers of renal function in diabetic and non-diabetic populations. Sci Rep. 2018;8 (1):15249. doi:10.1038/s41598-018-33507-7

40. Zhang S, Li X, Luo H, Fang ZZ, Ai H. Role of aromatic amino acids in pathogeneses of diabetic nephropathy in Chinese patients with type 2 diabetes. J Diabetes Complications. 2020;34(10):107667. doi:10.1016/j.jdiacomp.2020.107667

41. Du Y, Xu BJ, Deng X, et al. Predictive metabolic signatures for the occurrence and development of diabetic nephropathy and the intervention of Ginkgo biloba leaves extract based on gas or liquid chromatography with mass spectrometry. J Pharm Biomed Anal. 2019;166:30-39. doi:10.1016/j.jpba.2018.12.017

42. Woo CY, Baek JY, Kim AR, et al. Inhibition of ceramide accumulation in podocytes by myriocin prevents diabetic nephropathy. Diabetes Metab J. 2020;44(4):581-591. doi:10.4093/dmj.2019.0063

43. Tofte N, Suvitaival T, Ahonen L, et al. Lipidomic analysis reveals sphingomyelin and phosphatidylcholine species associated with renal impairment and all-cause mortality in type 1 diabetes. Sci Rep. 2019;9(1):16398. doi:10.1038/s41598-019-52916-w 
44. Sha Q, Lyu J, Zhao M, Li H, Guo M, Sun Q. Multi-omics analysis of diabetic nephropathy reveals potential new mechanisms and drug targets. Front Genet. 2020;11:616435. doi:10.3389/fgene.2020.616435

45. Niewczas MA, Sirich TL, Mathew AV, et al. Uremic solutes and risk of end-stage renal disease in type 2 diabetes: metabolomic study. Kidney Int. 2014;85(5):1214-1224. doi:10.1038/ki.2013.497

46. Chen CJ, Liao WL, Chang CT, Lin YN, Tsai FJ. Identification of urinary metabolite biomarkers of type 2 diabetes nephropathy using an untargeted metabolomic approach. J Proteome Res. 2018;17(11):3997-4007. doi:10.1021/acs.jproteome.8b00644

47. Peng L, Sun B, Liu Y, et al. Increased lipoxygenase and decreased cytochrome P450s metabolites correlated with the incidence of diabetic nephropathy: potential role of eicosanoids from metabolomics in type 2 diabetic patients. Clin Exp Pharmacol Physiol. 2021;48(5):679-685. doi:10.1111/1440-1681.13471

48. Niewczas MA, Mathew AV, Croall S, et al. Circulating modified metabolites and a risk of ESRD in patients with type 1 diabetes and chronic kidney disease. Diabetes Care. 2017;40(3):383-390. doi:10.2337/dc16-0173

49. Coughlan MT, Nguyen TV, Penfold SA, et al. Mapping time-course mitochondrial adaptations in the kidney in experimental diabetes. Clin Sci (Lond). 2016;130(9):711-720. doi:10.1042/CS20150838

50. Forbes JM, Thorburn DR. Mitochondrial dysfunction in diabetic kidney disease. Nat Rev Nephrol. 2018;14(5):291-312. doi:10.1038/nrneph.2018.9

51. Kimura T, Isaka Y, Yoshimori T. Autophagy and kidney inflammation. Autophagy. 2017;13(6):997-1003. doi:10.1080/15548627.2017.1309485

52. Ducasa GM, Mitrofanova A, Fornoni A. Crosstalk between lipids and mitochondria in diabetic kidney disease. Curr Diab Rep. 2019;19(12):144. doi:10.1007/s11892-019-1263-x

53. Hallan S, Afkarian M, Zelnick LR, et al. Metabolomics and gene expression analysis reveal down-regulation of the citric acid (TCA) cycle in non-diabetic CKD patients. EBioMedicine. 2017;26:68-77. doi:10.1016/j.ebiom.2017.10.027

54. Liu JJ, Liu S, Gurung RL, et al. Urine tricarboxylic acid cycle metabolites predict progressive chronic kidney disease in type 2 diabetes. $J$ Clin Endocrinol Metab. 2018;103(12):4357-4364. doi:10.1210/jc.2018-00947

55. Afshinnia F, Nair V, Lin J, et al. Increased lipogenesis and impaired beta-oxidation predict type 2 diabetic kidney disease progression in American Indians. JCI Insight. 2019;4(21):e31573977. doi:10.1172/jci.insight.130317

56. Davies EG, Petty RG, Kohner EM. Long term effectiveness of photocoagulation for diabetic maculopathy. Eye (Lond). 1989;3(6):764-767. doi:10.1038/eye.1989.119

57. Fouque D, Holt S, Guebre-Egziabher F, et al. Relationship between serum carnitine, acylcarnitines, and renal function in patients with chronic renal disease. J Ren Nutr. 2006;16(2):125-131. doi:10.1053/j.jrn.2006.01.004

58. Jarrell ZR, Smith MR, Hu X, et al. Plasma acylcarnitine levels increase with healthy aging. Aging (Albany NY). 2020;12(13):13555-13570. doi:10.18632/aging.103462

59. Marsden D, Bedrosian CL, Vockley J. Impact of newborn screening on the reported incidence and clinical outcomes associated with medium- and long-chain fatty acid oxidation disorders. Genet Med. 2021;23(5):816-829. doi:10.1038/s41436-020-01070-0

60. Rocha H, Castineiras D, Delgado C, et al. Birth prevalence of fatty acid beta-oxidation disorders in Iberia. JIMD Rep. 2014;16:89-94.

61. Almannai M, Alfadhel M, El-Hattab AW. Carnitine inborn errors of metabolism. Molecules. 2019;24(18):3251. doi:10.3390/molecules24183251

62. Giesbertz P, Ecker J, Haag A, Spanier B, Daniel H. An LC-MS/MS method to quantify acylcarnitine species including isomeric and odd-numbered forms in plasma and tissues. J Lipid Res. 2015;56(10):2029-2039. doi:10.1194/jlr.D061721

63. Xiang L, Wei J, Tian XY, et al. Comprehensive analysis of acylcarnitine species in db/db mouse using a novel method of high-resolution parallel reaction monitoring reveals widespread metabolic dysfunction induced by diabetes. Anal Chem. 2017;89(19):10368-10375. doi:10.1021/acs. analchem. $7 \mathrm{~b} 02283$

64. Mirzoyan K, Klavins K, Koal T, et al. Increased urine acylcarnitines in diabetic ApoE-/- mice: hydroxytetradecadienoylcarnitine (C14:2-OH) reflects diabetic nephropathy in a context of hyperlipidemia. Biochem Biophys Res Commun. 2017;487(1):109-115. doi:10.1016/j.bbrc.2017.04.026

65. Nkuipou-Kenfack E, Duranton F, Gayrard N, et al. Assessment of metabolomic and proteomic biomarkers in detection and prognosis of progression of renal function in chronic kidney disease. PLoS One. 2014;9(5):e96955. doi:10.1371/journal.pone.0096955

66. McCoin CS, Knotts TA, Adams SH. Acylcarnitines-old actors auditioning for new roles in metabolic physiology. Nat Rev Endocrinol. 2015;11 (10):617-625. doi:10.1038/nrendo.2015.129

67. Rebouche CJ. Kinetics, pharmacokinetics, and regulation of L-carnitine and acetyl-L-carnitine metabolism. Ann N Y Acad Sci. 2004;1033:30-41. doi:10.1196/annals.1320.003

68. Pande SV. A mitochondrial carnitine acylcarnitine translocase system. Proc Natl Acad Sci U S A. 1975;72(3):883-887. doi:10.1073/pnas.72.3.883

69. Guasch-Ferre M, Zheng Y, Ruiz-Canela M, et al. Plasma acylcarnitines and risk of cardiovascular disease: effect of Mediterranean diet interventions. Am J Clin Nutr. 2016;103(6):1408-1416. doi:10.3945/ajcn.116.130492

70. Yoshihisa A, Watanabe S, Yokokawa T, et al. Associations between acylcarnitine to free carnitine ratio and adverse prognosis in heart failure patients with reduced or preserved ejection fraction. ESC Heart Fail. 2017;4(3):360-364. doi:10.1002/ehf2.12176

71. McCann MR, George De la Rosa MV, Rosania GR, Stringer KA. L-carnitine and acylcarnitines: mitochondrial biomarkers for precision medicine. Metabolites. 2021;11(1):51. doi:10.3390/metabo11010051

72. Gunther SH, Khoo CM, Tai ES, et al. Serum acylcarnitines and amino acids and risk of type 2 diabetes in a multiethnic Asian population. BMJ Open Diabetes Res Care. 2020;8(1):e001315. doi:10.1136/bmjdrc-2020-001315

73. Aichler M, Borgmann D, Krumsiek J, et al. N-acyl taurines and acylcarnitines cause an imbalance in insulin synthesis and secretion provoking beta cell dysfunction in type 2 diabetes. Cell Metab. 2017;25(6):1334-1347. doi:10.1016/j.cmet.2017.04.012

74. Muilwijk M, Goorden SMI, Celis-Morales C, et al. Contributions of amino acid, acylcarnitine and sphingolipid profiles to type 2 diabetes risk among South-Asian Surinamese and Dutch adults. BMJ Open Diabetes Res Care. 2020;8(1):e001003. doi:10.1136/bmjdrc-2019-001003

75. Limonte CP, Valo E, Montemayor D, et al. A targeted multiomics approach to identify biomarkers associated with rapid eGFR decline in type 1 diabetes. Am J Nephrol. 2020;51(10):839-848. doi:10.1159/000510830

76. Fort PE, Rajendiran TM, Soni T, et al. Diminished retinal complex lipid synthesis and impaired fatty acid beta-oxidation associated with human diabetic retinopathy. JCI Insight. 2021;6(19):e152109. doi:10.1172/jci.insight.152109

77. Li X, Li Y, Liang Y, Hu R, Xu W, Liu Y. Plasma targeted metabolomics analysis for amino acids and acylcarnitines in patients with prediabetes, type 2 diabetes mellitus, and diabetic vascular complications. Diabetes Metab J. 2021;45(2):195-208. doi:10.4093/dmj.2019.0209 
78. Zhao S, Feng XF, Huang T, et al. The association between acylcarnitine metabolites and cardiovascular disease in Chinese patients with type 2 diabetes mellitus. Front Endocrinol (Lausanne). 2020;11:212. doi:10.3389/fendo.2020.00212

79. Xia FY, Zhu L, Xu C, et al. Plasma acylcarnitines could predict prognosis and evaluate treatment of IgA nephropathy. Nutr Metab (Lond). 2019;16:2. doi:10.1186/s12986-018-0328-1

80. Afshinnia F, Rajendiran TM, Soni T, et al. Impaired beta-oxidation and altered complex lipid fatty acid partitioning with advancing CKD. J Am Soc Nephrol. 2018;29(1):295-306. doi:10.1681/ASN.2017030350

81. Thongnak L, Pongchaidecha A, Lungkaphin A. Renal lipid metabolism and lipotoxicity in diabetes. Am J Med Sci. 2020;359(2):84-99. doi:10.1016/j.amjms.2019.11.004

82. Bremer J. Carnitine as a fatty acid carrier in intermediary metabolism. Nature. 1962;196(4858):993-994. doi:10.1038/196993a0

83. Hocher B, Adamski J. Metabolomics for clinical use and research in chronic kidney disease. Nat Rev Nephrol. 2017;13(5):269-284. doi:10.1038/ nrneph.2017.30

84. van der Kloet FM, Tempels FW, Ismail N, et al. Discovery of early-stage biomarkers for diabetic kidney disease using ms-based metabolomics (FinnDiane study). Metabolomics. 2012;8(1):109-119. doi:10.1007/s11306-011-0291-6

85. Chuang W-H, Arundhathi A, Lu C, et al. Altered plasma acylcarnitine and amino acid profiles in type 2 diabetic kidney disease. Metabolomics. 2016;12(6):108. doi:10.1007/s11306-016-1049-y

86. Khamis MM, Adamko DJ, El-Aneed A. Mass spectrometric based approaches in urine metabolomics and biomarker discovery. Mass Spectrom Rev. 2017;36(2):115-134. doi:10.1002/mas.21455

87. Lains I, Gantner M, Murinello S, et al. Metabolomics in the study of retinal health and disease. Prog Retin Eye Res. 2019;69:57-79. doi:10.1016/j. preteyeres.2018.11.002

88. Jang C, Chen L, Rabinowitz JD. Metabolomics and isotope tracing. Cell. 2018;173(4):822-837. doi:10.1016/j.cell.2018.03.055

89. Rebholz CM, Zheng Z, Grams ME, et al. Serum metabolites associated with dietary protein intake: results from the Modification of Diet in Renal Disease (MDRD) randomized clinical trial. Am J Clin Nutr. 2019;109(3):517-525. doi:10.1093/ajen/nqy202

90. Hirschel J, Vogel M, Baber R, et al. Relation of whole blood amino acid and acylcarnitine metabolome to age, sex, BMI, puberty, and metabolic markers in children and adolescents. Metabolites. 2020;10(4):149. doi:10.3390/metabo10040149

91. Rinschen MM, Ivanisevic J, Giera M, Siuzdak G. Identification of bioactive metabolites using activity metabolomics. Nat Rev Mol Cell Biol. 2019;20(6):353-367. doi:10.1038/s41580-019-0108-4

92. Chen KH, Chen YL, Tang HY, et al. Dietary leucine supplement ameliorates hepatic steatosis and diabetic nephropathy in db/db mice. Int $J$ Mol Sci. 2018;19(7):1921. doi:10.3390/ijms19071921

93. Quan W, Jiao Y, Xue C, et al. The effect of exogenous free N(epsilon)-(Carboxymethyl)lysine on diabetic-model Goto-Kakizaki rats: metabolomics analysis in serum and urine. J Agric Food Chem. 2021;69(2):783-793. doi:10.1021/acs.jafc.0c06445

94. Zahoor I, Rui B, Khan J, Datta I, Giri S. An emerging potential of metabolomics in multiple sclerosis: a comprehensive overview. Cell Mol Life Sci. 2021;78(7):3181-3203. doi:10.1007/s00018-020-03733-2

Diabetes, Metabolic Syndrome and Obesity: Targets and Therapy

\section{Publish your work in this journal}

Diabetes, Metabolic Syndrome and Obesity: Targets and Therapy is an international, peer-reviewed open-access journal committed to the rapid publication of the latest laboratory and clinical findings in the fields of diabetes, metabolic syndrome and obesity research. Original research, review, case reports, hypothesis formation, expert opinion and commentaries are all considered for publication. The manuscript management system is completely online and includes a very quick and fair peer-review system, which is all easy to use. Visit http://www.dovepress. com/testimonials.php to read real quotes from published authors.

Submit your manuscript here: https://www.dovepress.com/diabetes-metabolic-syndrome-and-obesity-targets-and-therapy-journal 